\title{
Prediction for Risk of Recurrence and Delayed Metastases of Nasopharyngeal Carcinoma: An evaluation of CT scan
}

\author{
Umasan Phuprasat, M.D. \\ Anchalee Churojana, M.D. \\ Kullathorn Thepmongkol, M.D.
}

Division of General Radiology, Department of Radiology, Faculty of Medicine, Siriraj Hospital, Mahidol University,

2 Prannok Road, Bangkok Noi, Bankok, Thailand 10700

\begin{abstract}
Purpose: To analyze the imaging characteristic on CT scan of the primary nasopharyngeal carcinoma which predict the locoregional recurrence.

Materials and Methods: A retrospective review of 464 patients who had nasopharyngeal carcinoma at Siriraj Hospital, during 2004-2009 was performed. The treatment options were radiation therapy and concurrent chemotherapy corresponding to the staging, which were assessed by using CT. The patients who had no available initial imaging studies, incomplete treatment or had follow up less than 1 year were excluded. The imaging studies were analyzed for primary tumor size, direct tumor extension, and cervical lymph node involvement. The vascular invasion was defined as the obliteration more than half of the circumferential fat surrounding internal carotid artery. The regional nodes were evaluated for the levels of involvement and size of the largest one.

Results: There were 99 patients (68 male, 31 female, mean age at 50 years) with the TNM classifications as follows: T15.2\%, T2 39.4\%, T3 24.2\%, T4 21.2\%, N0 18.2\%, N1 14.1\%, N2 46.5\% and metastasis $11.76 \%$. The extensions were prevertebral space $(54.5 \%)$, masticator space $(30.3 \%)$, skull base destruction (18.2\%), and vascular invasion (42.4\%). Nodal involvement was found in $81.8 \%$. The most frequency of the largest lymph node was at ipsilateral level V (38.4\%) and II (41.4\%). Recurrence was found in $40.4 \%$ with averaged at 24 months after complete treatment $(95 \% \mathrm{Cl})$. Vascular invasion and skull base invasion had significant associated to predictive the risk of recurrence ( $\mathrm{P}=0.04$ and $<0.001$, respectively). The size of the tumor or lymph node showed no relation to the local regional recurrence or metastasis.

Conclusion: The risks of NPC recurrence have been correlated to TNM staging, skull base, and carotid space involvement. Skull base invasion has been identified to be the predictive sign for tumor recurrence. Skull base invasion and carotid space involvement have also a statistically significant prognostic factor for overall survival.
\end{abstract}




\section{Introduction}

Nasopharyngeal carcinoma (NPC) is an aggressive cancer which is endemic in Southern China and South East Asia, which has the incidence accounting 2-10 per 100,000 person-years ${ }^{1-3}$. The gross mortality of NPC is reported about 1.34 per 100 000 , comprises $1.61 \%$ of tumor caused deaths and ranks ninth in China ${ }^{4-5}$.

In Thailand, according to the statistics database of Siriraj Cancer Institute Registry in 2008, new cases of NPC were accounted for $3.28 \%$ and $1.21 \%$ which were ranking at the eighth and thirteenth common cancer in male and female patients, respectively ${ }^{6}$.

Although NPC is very radiosensitive and the treatment outcome have improved in the recent decades, but rate of local regional failure and tumor recurrence can occur in 19\%-56\% of patients within 2-5 years after definite radiotherapy ${ }^{4,5,7,8}$. The prognosis following local recurrence is rather poor without retreatment ${ }^{8,9}$. Moreover, after treatment, clinical examination for detection of residual or recurrence may be inconclusive; imaging studies may be equivocal due to the presence of diffuse soft tissue edema. In such cases, tumor recurrence should be confirmed by biopsy ${ }^{4.5}$.

Thus, it is essential to be able to predict the therapeutic response of the patients with NPC from the initial imaging study which CT scan is widely common used ${ }^{4,8-10}$. Those patients may be need more closely follow up for the early detection of tumor recurrence. In this regard, early retreatment may be benefit to achieve better tumor control and improve patient survival ${ }^{4,5,7-10}$.

The purpose of this paper is to analyze whether any characteristics or sign on initial CT scan which may predict the risk of tumor recurrence in the patients with NPC after receiving complete treatment.

\section{Materials and Methods}

\section{Patient characteristics}

A number of 464 patients who had pathological proven of nasopharyngeal carcinoma (NPC) at Siriraj Hospital, Mahidol University, between January 2004 and April 2009 were retrospectively reviewed, under the approval of the ethic committee of our hospital.

All patients received definite standard radiation treatment with or without concurrent chemotherapy corresponding to their own primary stage. Our radiation therapy protocol for primary NPC was achieved from external-beam radiation, either conventional radiotherapy or integrated moderator radiotherapy (IMRT) at a mean dose of 70 Gy in 33 fractions (range, 65-80 Gy), which were 1.8 to 2.0 Gy per fraction for 5 fractions at a weekly interval.

After complete treatment, the patients had scheduled to follow up with evaluation of the treatment response by the joint clinics of otolaryngology, radiotherapy, and oncology for every 6-8 weeks in the first year, every 3 months in the second year, once every 4-6 months in the third year, and once every 6-9 months, thereafter. Endoscopic examination of the nasopharynx was evaluated at 6-8 weeks. The follow-up imaging was performed within 3-12 months or whenever there was clinical suspicion of tumor recurrence.

\section{The exclusion criterias were included}

1. The patients who had unavailable initial imaging study.

2. The patients who had incomplete treatment or loss to follow up.

3. The patients who had less than 12 months 
follow up after complete treatment.

4. The patients who had history of other underlying malignancy with cervical lymph node metastasis.

5. The patients who had advanced staging or distant metastasis at the presenting diagnosis which included intracranial extension, and/or metastases to mediastinal lymphnode, lung, skeleton, liver, and miscellaneous (bone marrow, skin, other viscera and soft tissue).

6. The patients who had progressive disease or distant metastasis during or immediate after complete treatment.

\section{Tumor response and recurrence}

The patients who were enrolled in this study were divided into 2 groups; recurrent and non recurrent groups.

The recurrent group included those patients who had increase or newly detectable one or more of the following lesions; 1. loco-regional or primary site, 2. cervical lymph node, 3. distant metastases during the follow up period for at least one year, after initial document of tumor free-period or at least stable disease after complete treatment. The recurrent disease was diagnosed by information of physical examination, endoscopic findings, imaging feature and histopathology.

The non-recurrent group was defined as the patients who had responsed to the treatment or at least stable disease after complete treatment without evidence of new lesions or recurrence over one year period.

The patients who had progressive disease after treatment, either at the primary site or cervical lymph nodes were excluded from the study.

\section{CT scan analysis}

The initial CT scans were reviewed by one neuroradiologist who had more than 10 years experience and blinded from the follow up outcome of the patients. The CT images were retrieved from the picture archiving and communication system (PACS) for evaluation of the primary tumor in term of side, size (averaged diameter), extension and tumor staging using TNM staging according to AJCC 2002 guideline ${ }^{11}$. The side was observed to be one or both sides of tumor involvement. The tumor size was calculated in the averaged diameter which measured in two maximal dimensions with perpendicular axes to each other on the cross sectional CT image (figure 1) and classified as less than $5 \mathrm{~mm}, 5-10 \mathrm{~mm}, 11-15 \mathrm{~mm}$ and more than $15 \mathrm{~mm}$. The direct extension of the primary tumors are categorized into many regions as parapharyngeal space, masticator space, parotid space, prevertebral space, skull base, internal carotid artery (ICA) or vascular invasion and presence of retropharyngeal lymph nodes.

Our criteria for ICA invasion was at least demonstration of obliteration of the circumferential fat plane between ICA and tumor more than 180 degrees.

The cervical lymph nodes were interpreted as the level system according to 1977 AJCC nodal classification $^{12}$. However, the supraclavicular group was named separately. They were documented as positive if the short axis was longer than $10 \mathrm{~mm}$.

\section{Statistical analysis}

All the statistical analyses in this study were carried out using SPSS for Windows software version 13.0 (SPSS; statistical software version 13.0). 


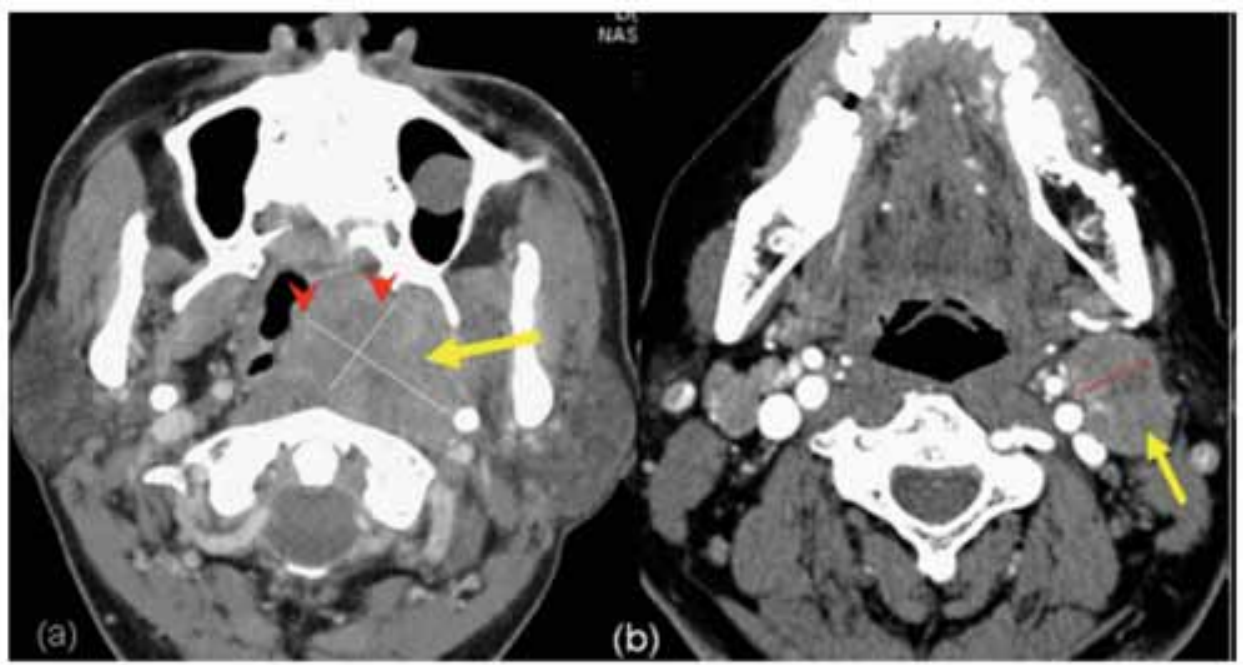

Fig. 1 Demonstrated of primary tumor and lymph node measurements

(a) The primary tumor (arrow) measurement on contrast enhanced CT image in the axial plane was obtained by the averaged measurement in two greatest dimensions in perpendicularly axes to each other (arrow head) and classified into 4 groups as less than $5 \mathrm{~mm}, 5-10 \mathrm{~mm}$, 11-15 $\mathrm{mm}$ and more than $15 \mathrm{~mm}$.

(b) The regional nodal measurement was performed by single maximal diameter in short axis.

The univariate analysis was using the log rank test and multivariate analysis was using the Cox proportional hazards model. Survival analysis were performed using estimation method of Kaplan and Meier. Overall survival (OS) in this study was defined as the time from NPC diagnosis to death resulting from any cause or at the time of last follow-up. $P$ values less than 0.05 was considered statistically significant.

\section{Results}

Of 464 patients with NPC, there were 99 patients (68 men, and 31 women) who met our criteria. All patients had mean time of follow up at 24 months. Recurrent disease was found in 40/99 $(40.4 \%)$ including locoregional recurrence at primary site and/or cervical lymph node in 19 cases (19.2\%) and distance metastasis in 21 cases $(21.2 \%)$, with a relapse time range from 6 to 58 months (mean 12 months) after complete treatment. The comparison of the demographic data and tumor characteristics of recurrent and non recurrent groups were shown in table 1, which showed no significant statistically difference between both groups.

Concerning characteristics of primary lesion of NPC (table 2), the T stage of primary tumor according to AJCC 2002 TNM classification had statistically significant correlation to risk of tumor recurrence $(P=0.044)$, whereas the higher $T$ stage had higher risk of recurrence. The lateralization and size of the primary tumor showed no statistically significant to predict risk of recurrence of NPC.

With respect to direct extension of the NPC to other neck spaces (table 3), there were significant association of tumor recurrence with the involvement of carotid space or vascular invasion 
Table 1: Patient and tumor characteristics of recurrence and non recurrence groups

\begin{tabular}{|c|c|c|c|}
\hline \multirow[b]{2}{*}{ Character } & \multirow{2}{*}{$\begin{array}{c}\text { Total } \\
n=99\end{array}$} & \multicolumn{2}{|c|}{ Number of patients } \\
\hline & & $\begin{array}{c}\text { Recurrent } \\
n=40\end{array}$ & $\begin{array}{c}\text { Non recurrent } \\
n=59\end{array}$ \\
\hline Sex: Men & 68 & $30(44.12 \%)$ & $38(55.88 \%)$ \\
\hline Women & 31 & $10(29.03 \%)$ & $21(70.96 \%)$ \\
\hline Age range ( Mean age: years) & $18-82(50.23)$ & $18-74(48.61)$ & $33-82(50.30)$ \\
\hline \multicolumn{4}{|l|}{ Pathological Feature (WHO) } \\
\hline Type I: NK,SCC & 11 & $4(36.36 \%)$ & $7(63.64 \%)$ \\
\hline Type II: SCC(mod-poor diff) & 45 & $21(46.66 \%)$ & $24(53.33 \%)$ \\
\hline Type III: Undifferentiated, SCC & 43 & $15(34.88 \%)$ & $28(65.12 \%)$ \\
\hline \multicolumn{4}{|l|}{ Treatment } \\
\hline Definite Radiation Therapy & 4 & $1(25 \%)$ & $3(75 \%)$ \\
\hline Concurrent chemoradiation & 95 & $55(55.56 \%)$ & $44(44.44 \%)$ \\
\hline Mean radiation dose & $68 \pm 2$ Grey & $68 \pm 2$ Grey & $68 \pm 2$ Grey \\
\hline Mean time of follow up (months) & $24+2$ & $24+2$ & $24+2$ \\
\hline
\end{tabular}

Table 2: Comparison of feature of primary NPC between recurrence and non recurrence groups

\begin{tabular}{|c|c|c|c|c|}
\hline \multirow[b]{2}{*}{ Parameter } & \multirow[b]{2}{*}{$\begin{array}{c}\text { Total Number } \\
n=99 \\
(100 \%)\end{array}$} & \multicolumn{2}{|c|}{ Number of patients } & \multirow[b]{2}{*}{$p$-value } \\
\hline & & $\begin{array}{c}\text { Recurrent group } \\
n=40 \\
(40.4 \%)\end{array}$ & $\begin{array}{c}\begin{array}{c}\text { No recurrent } \\
\text { group } \\
n=59(59.6 \%)\end{array}\end{array}$ & \\
\hline \multicolumn{5}{|l|}{ Location $^{\text {a }}$ of NPC } \\
\hline One side of NP & $69(70)$ & $24(63.13)$ & $31(36.87)$ & 0.452 \\
\hline Both side of NP & $30(30)$ & $16(53.33)$ & $14(46.67)$ & \\
\hline \multicolumn{5}{|l|}{ Primary tumor size ${ }^{b}$} \\
\hline$<5 \mathrm{~mm}$ & $6(6.06)$ & $4(66.67)$ & $2(33.33)$ & 0.385 \\
\hline $5-10 \mathrm{~mm}$ & $5(5.05)$ & $2(40.00)$ & $3(60.00)$ & \\
\hline $11-15 \mathrm{~mm}$. & $13(13.13)$ & $7(53.85)$ & $6(46.15)$ & \\
\hline$>15 \mathrm{~mm}$ & $75(75.76)$ & $27(36.00)$ & $48(64.00)$ & \\
\hline \multicolumn{5}{|l|}{ TNM Classification $^{\circ}$} \\
\hline T stage: $\mathrm{T} 1$ & $15(15.15)$ & $7(46.67)$ & $8(53.33)$ & 0.044 \\
\hline T2 & 39 (39.39) & $7(17.95)$ & $32(82.05)$ & \\
\hline T3 & $24(24.24)$ & $14(58.33)$ & $10(41.67)$ & \\
\hline $\mathrm{T} 4$ & $21(21.21)$ & $12(57.14)$ & $9(42.86)$ & \\
\hline
\end{tabular}

Using Cox regression Analysis and significant p-value was less than 0.05

$\mathrm{n}$ : Number of cases in each group

a: Lateralization of primary nasopharyngeal cancer (NPC) which was stratified as involved one side of NP included midline lesion, or involved both side of NP

b: Primary tumor size measured from initial CT image

c: TNM tumor classification according to AJCC 2002 guideline 
January-April 2013, Volume XIX No.I

Table 3: Comparison of tumor extension between recurrence and non recurrence groups

\begin{tabular}{|ccccc|}
\hline & & \multicolumn{2}{c}{ Number of patients } & \\
\cline { 3 - 4 } Direction of NPC & Total & Recurrent group & No recurrent & p-value \\
spreading & $\mathrm{n}=99$ & $\mathrm{n}=40$ & Group $\mathrm{n}=59$ & \\
& $(100 \%)$ & $(40.4 \%)$ & $(59.6 \%)$ & \\
Parapharyngeal space & $22 / 99$ & 8 & 14 & 0.41 \\
invasion & $(22.22)$ & $(36.36)$ & $(63.64)$ & \\
Masticator space invasion & $30 / 99$ & 13 & 17 & 0.40 \\
Prevertebral space & $(33.33)$ & $(43.33)$ & $(56.67)$ & \\
invasion & $54 / 99$ & 20 & 34 & 0.39 \\
Parotid space invasion & $(54.55)$ & $(37.04)$ & $(62.96)$ & 0.56 \\
Vascular invasion & $(7 / 99$ & 4 & 3 & $0.04{ }^{1}$ \\
Skull base invasion & $42 / 99$ & $(57.14)$ & $(42.86)$ & \\
& $(42.42)$ & $(54.76)$ & $(45.24)$ & $0.001^{2}$ \\
\hline
\end{tabular}

Using Cox regression analysis and significant $p$-value was less than 0.05

1: The hazard risk was 2.05 with $95 \%$ Confidential Interval 1.03-4.06.

2: The hazard risk was 0.26 with $95 \%$ Confidential Interval $=0.13-0.52$.

Table 4: Nasopharyngeal carcinoma: $6^{\text {th }}$ edition TNM Classification (2002) according to AJCC staging system guideline ${ }^{11}$

\begin{tabular}{|c|c|}
\hline \multicolumn{2}{|r|}{ T stage } \\
\hline T1 & Tumor confined to nasopharynx \\
\hline T2 & Tumor extends to soft tissue \\
\hline T2a & Tumor extends to oropharynx and/or nasal cavity without parapharyngeal extension \\
\hline $\mathrm{T} 2 \mathrm{~b}$ & Any tumor with parapharyngeal extension \\
\hline T3 & Tumor invades bony structures and/or paranasalsinuses \\
\hline T4 & $\begin{array}{l}\text { Tumor with intracranial extension and/or involvement of cranial nerves, } \\
\text { infratemporal fossa, hypopharynx, orbit, or masticator space }\end{array}$ \\
\hline \multicolumn{2}{|r|}{$\mathrm{N}$ stage } \\
\hline NO & No regional lymph node ( $\mathrm{LN})$ metastasis \\
\hline N1 & $\begin{array}{l}\text { Unilateral metastasis in LN (s), } \leq 6 \mathrm{~cm} \text { in greatest dimension, } \\
\text { above supraclavicular fossa }\end{array}$ \\
\hline N2 & $\begin{array}{l}\text { Bilateral metastasis in LN (s), } \leq 6 \mathrm{~cm} \text { in greatest dimension, } \\
\text { above supraclavicular fossa }\end{array}$ \\
\hline N3 & Metastasis in LN (s) $>6 \mathrm{~cm}$ and/or to supraclavicular fossa \\
\hline N3a & Metastasis in $\mathrm{LN}(\mathrm{s})>6 \mathrm{~cm}$ in dimension \\
\hline N3b & Metastasis in LN (s) with extension to supraclavicular fossa \\
\hline \multicolumn{2}{|r|}{ Stage group } \\
\hline I & T1NOMO \\
\hline IIA & T2aNOMO \\
\hline IIB & T2bNOMO or T1-T2N1MO \\
\hline III & T1-T3N2MO or T3NO-N1MO \\
\hline IVA & T4NO-N2MO \\
\hline IVB & Any $\mathrm{T}$ N3, MO \\
\hline IVC & Any $\mathrm{T}$, any $\mathrm{N}, \mathrm{M} 1$ \\
\hline
\end{tabular}


Table 5: Comparison of the feature of lymph nodes between recurrent and non recurrent groups

\begin{tabular}{|c|c|c|c|c|}
\hline \multirow[b]{2}{*}{ Parameter } & \multirow{2}{*}{$\begin{array}{c}\text { Total } \\
\mathrm{n}=99 \\
(100 \%)\end{array}$} & \multicolumn{2}{|c|}{ Number of patients } & \multirow{2}{*}{$p$-value } \\
\hline & & $\begin{array}{l}\text { Recurrent group } \\
n=40(40.4 \%)\end{array}$ & $\begin{array}{c}\text { No recurrent } \\
\mathrm{n}=59(59.6 \%)\end{array}$ & \\
\hline N stage : NO & $18(18.2)$ & $5(27.78)$ & $13(72.22)$ & 0.002 \\
\hline N1 & $14(14.1)$ & $10(71.42)$ & $4(28.57)$ & \\
\hline N2 & $46(46.5)$ & $13(28.26)$ & $33(71.74)$ & \\
\hline N3 & $21(21.2)$ & $12(57.14)$ & $9(42.86)$ & \\
\hline \multicolumn{5}{|l|}{ Cervical nodes involvement } \\
\hline Ipsilateral side of NPC & $59(59.6)$ & $23(38.98)$ & $36(61.01)$ & 0.690 \\
\hline Contralateral side NPC & $40(40.4)$ & $17(42.50)$ & $23(57.50)$ & \\
\hline \multicolumn{5}{|l|}{ Level of nodal involvement } \\
\hline Cervical LN group II & $41(41.4)$ & $27(65.8)$ & $14(34.2)$ & 0.954 \\
\hline Cervical LN group III & $5(5.1)$ & $3(60.0)$ & $2(40.0)$ & \\
\hline Cervical LN group IV & $1(1.0)$ & $0(0.0)$ & $1(100)$ & \\
\hline Cervical LN group $\vee$ & $38(38.4)$ & $20(52.6)$ & $18(47.4)$ & \\
\hline All II - V levels & $8(8.1)$ & $5(62.5)$ & $3(37.5)$ & \\
\hline RPLN & $39(39.4)$ & $19(48.7)$ & $20(51.3)$ & \\
\hline SPC & $6(6.1)$ & $4(66.7)$ & $2(33.3)$ & \\
\hline \multicolumn{5}{|l|}{ Maximal diameter of LN } \\
\hline Less than $1.0 \mathrm{~cm}$ & $17(17.2)$ & $4(23.5)$ & $13(76.5)$ & 0.064 \\
\hline $1.0-1.5 \mathrm{~cm}$ & $25(25.3)$ & $6(6.1)$ & 19 (93.9) & \\
\hline$>1.5-2.0 \mathrm{~cm}$ & $20(20.2)$ & $11(55.0)$ & $9(45.0)$ & \\
\hline$>2.0 \mathrm{~cm}$ & $24(24.2)$ & $11(45.8)$ & $13(54.2)$ & \\
\hline Matted Iymph node & $13(13.1)$ & $8(61.5)$ & $5(38.5)$ & \\
\hline (Averaged diameter of LN) & - & $3.6 \mathrm{~cm}$ & $1.5 \mathrm{~cm}$ & 0.178 \\
\hline
\end{tabular}

Table 6: Multivariate Cox regression analysis of significant factors

\begin{tabular}{|c|c|c|c|c|}
\hline \multirow{4}{*}{ Predictive Factors } & Recurrent & No recurrent & \multirow{4}{*}{$P$-values* } & \multirow[b]{2}{*}{$\mathrm{HR}^{1}$} \\
\hline & NPC & NPC & & \\
\hline & $n=40$ & $n=59$ & & {$[95 \% \mathrm{Cl}]$} \\
\hline & $(40.4 \%)$ & $(59.6 \%)$ & & \\
\hline \multirow[t]{2}{*}{ Primary T stage } & $40 / 99$ & $59 / 99$ & 0.637 & - \\
\hline & $(40.4)$ & $(59.6)$ & & \\
\hline \multirow[t]{2}{*}{ Primary N stage } & $40 / 99$ & $59 / 99$ & 0.657 & - \\
\hline & $(40.4)$ & $(59.6)$ & & \\
\hline \multirow[t]{2}{*}{ Vascular invasion } & $23 / 99$ & $19 / 99$ & 0.285 & - \\
\hline & $(23.23)$ & (19.19) & & \\
\hline \multirow[t]{2}{*}{ Skull base invasion } & $14 / 99$ & $4 / 99$ & $<0.001$ & 0.259 \\
\hline & $(14.14)$ & $(4.04)$ & & [0.13-0.59] \\
\hline
\end{tabular}

*Using Cox regression analysis and significant $p$-value was less than 0.05

1 Harzard Risk (HR) value of Cox regression analysis 
[P-value $=0.04$ and $95 \% \mathrm{Cl} ; 1.03-4.06, \mathrm{HR}=2.05]$ and skull base extension $[\mathrm{P}$-value $=<0.001$ and $95 \% \mathrm{Cl}$; 0.13-0.52, HR=0.26]. In our study, extension to other adjacent spaces such as parapharyngeal space, masticator space or parotid space had no correlation with the risk of recurrence.

All patients had cervical lymph node enlargement at presentation. The distribution of lymph nodes which were classified according to level system, the nodal stage according to AJCC 2002 TNM classification and nodal size were demonstrated in table 4 and 5 . According to our data, the only lymph node feature that had statistically significant to associate with the risk of recurrent NPC was correlated with nodal stage of TNM classification ( $P=0.007)$. Similar to $T$ stage, the risk of recurrence was increased with the higher $\mathrm{N}$ stage. In our study, the mean nodal size was $3.6 \mathrm{~cm}$ in the short axis of recurrent group whereas it was $1.5 \mathrm{~cm}$ in the non recurrent group. However, there was no statistically significant to prognostic risk of recurrence between these 2 groups. ( $P=0.064)$.

However, after using multivariate analysis by Cox proportion and hazard model of statistically significant factors (as shown in table 6), which were associated with tumor recurrence, including primary $\mathrm{T}$ and $\mathrm{N}$ stage, vascular invasion and skull base invasion. The result showed only skull base invasion that is persistent statistically significant feature $(P<0.001)$, whereas another features showed no statistically significant $(P>0.05)$. Thus, the skull base invasion was a predictive sign to determine risk of recurrent NPC and another feature was undetermined.

In our study, twenty-one of 40 patients with recurrent disease had distant metastases, 13 pa- tients was found metastases to lung, 7 to liver, 3 to skeleton, and 2 had subcutaneous nodules, which the further tissues biopsy showed metastasis carcinoma. Three of them had both lung and bony metastases and one of them had progressive liver involvement after lung metastasis at the time of last follow up. The interval duration from post complete treatment to metastases was averaged at 24 months.

When correlation between the follow up time and evidence of recurrent NPC, overall survival rate for all 99 NPC patients were $85 \%, 71 \%, 56 \%$, and $50 \%$ in first, second, third, and fourth-year after diagnosis, respectively (figure 2). The median overall survival rate was 39 months.

Apart of direct extension of the NPC to other neck spaces, there were two spaces of direct extension that had profound influence of the patient's survival rate. The first one was a vascular invasion (figure 3); the patient who had a vascular invasion will be the time of recurrence NPC rapidly more than the patient, who did not have the vascular invasion. Median time of recurrence was 34 months in patient with vascular invasion and 43 months in patient without this feature ( $P$ value $=0.032$ )

Similarly to survival time analysis of skull base invasion (figure 4); the patient who had a skull base invasion will have recurrence more rapidly than the patient who did not have the skull base invasion. Median time of recurrence were 24 and 44 months in patient with and without skull base invasion, respectively ( $P$ value $=<0.001$ )

In another parameter, included primary tumor size, location, the rest of primary extension, nodal size, and nodal levels, had similarly in median time of recurrence and no statistically significant difference within groups. 
Fig. 2 Overall survival rate of patient's entire group at mean of covariates $(n=99)$.

\section{Survival Function atmean of covariates}

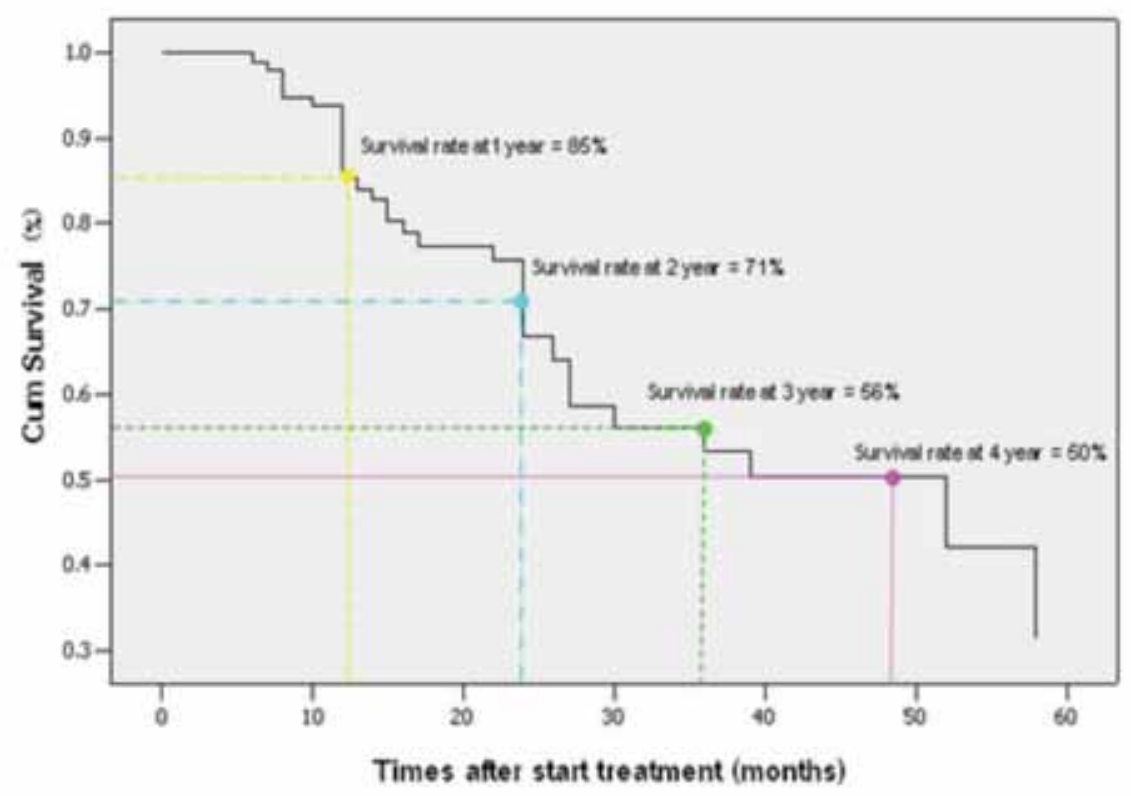

Fig. 3 Survival of patients for the different levels of vascular/carotid space invasion Survival Functions

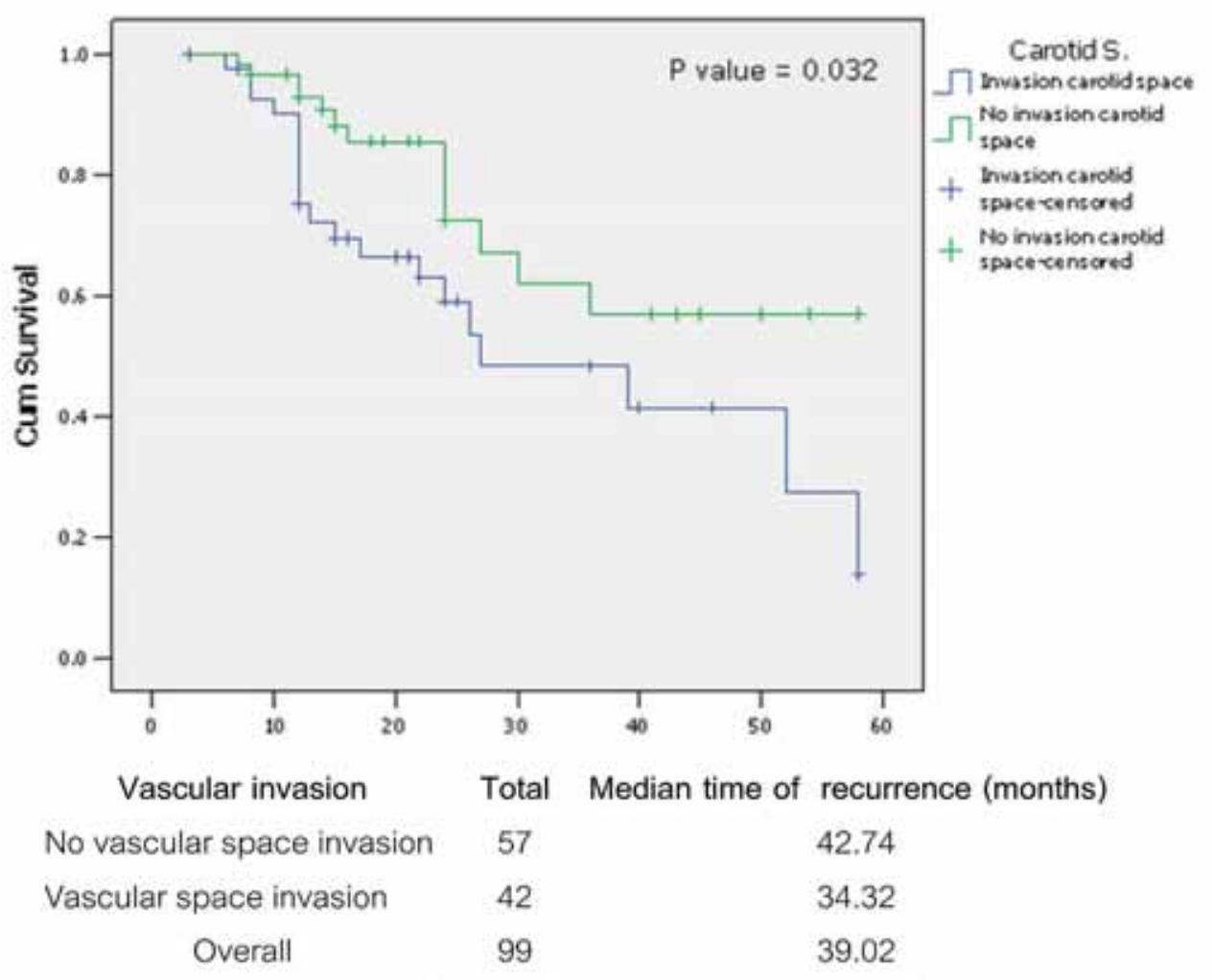

Kaplan-Meier analysis was defined significant $p$ value less than 0.005 
Fig. 4 Survival of patients by skull base invasion

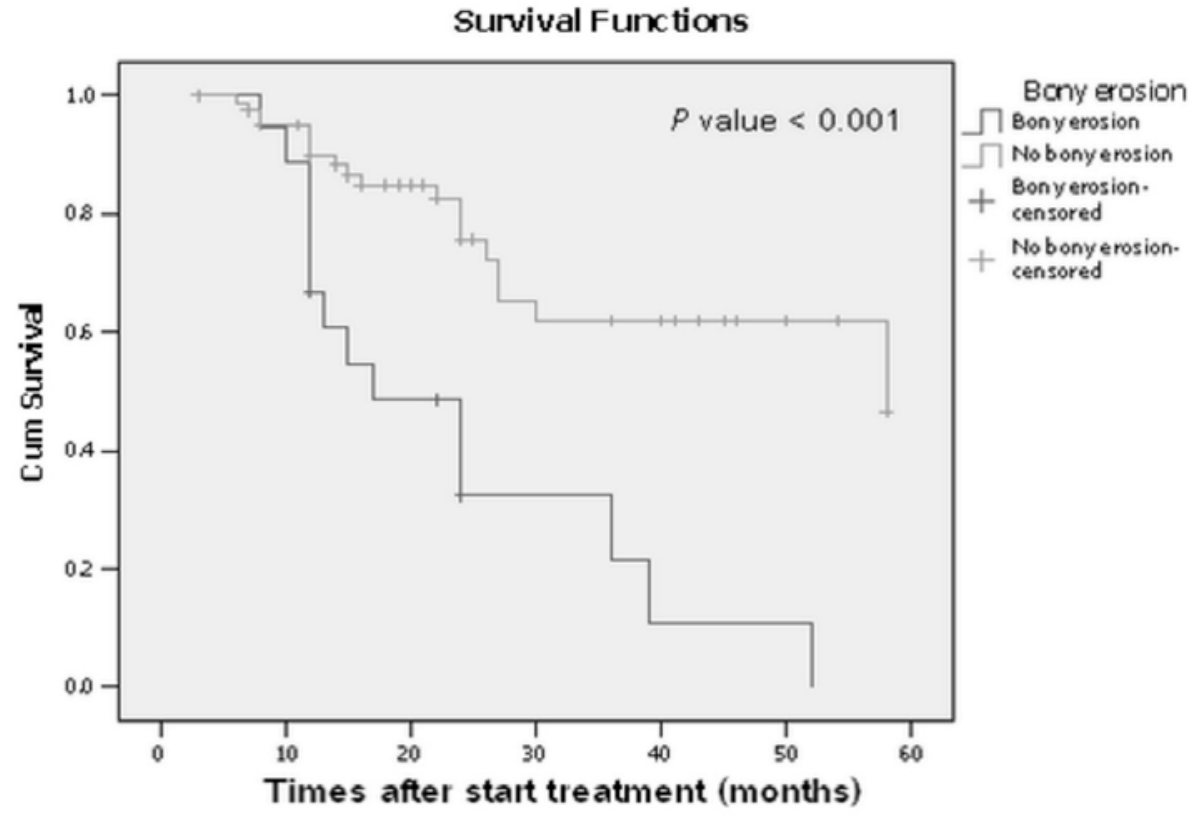

Bony skull invasion Total Median time of recurrence (months)

$\begin{array}{ccc}\text { No bony skull invasion } & 81 & 43.564 \\ \text { Bony skull invasion } & 18 & 23.956 \\ \text { Overall } & 99 & 39.017\end{array}$

Kaplan-Meier analysis was defined significant $p$ value less than 0.005

Fig. 5 Vascular or Carotid space invasion

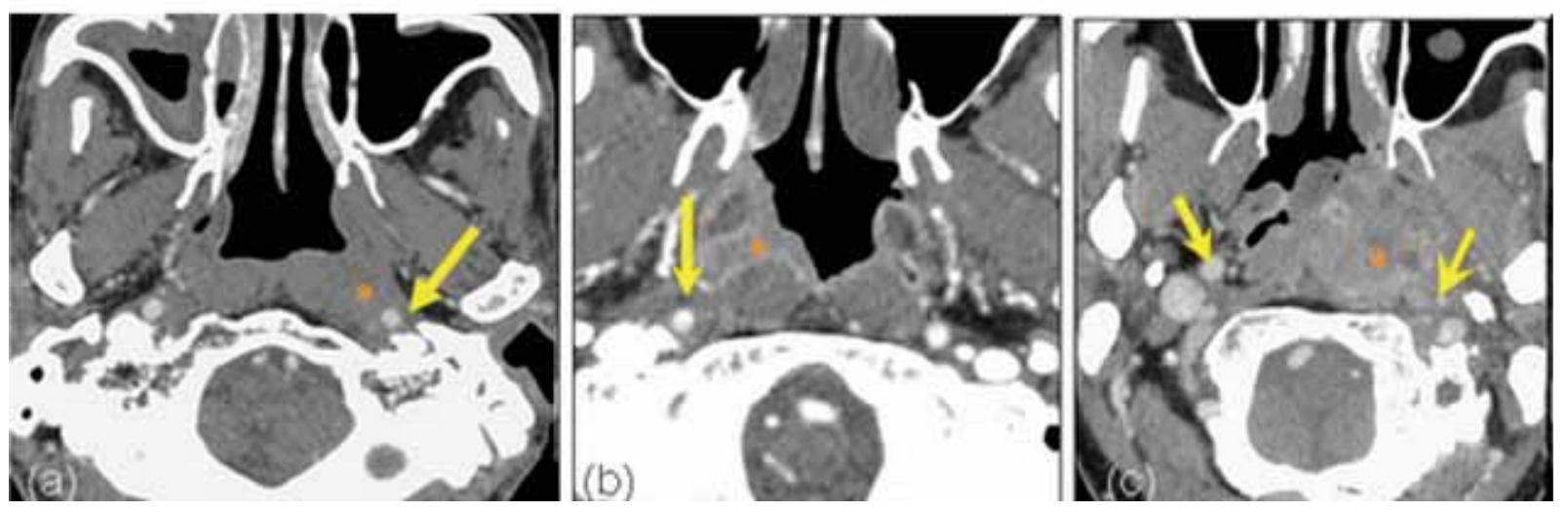

(a) Axial contrast-enhanced CT scan of the nasopharynx in a patient with NPC. There is an enhancing tumor at left nasopharynx $\left(^{*}\right)$, which extension caused obliterate the circumferential fat plane of left internal carotid artery or internal jugular vein more than half of diameter (arrow). Comparisons to the right sided, which preserves normal fat plane.

(b) Axial contrast-enhanced CT scan of the nasopharynx in a different patient with NPC. There is an enhancing tumor at right nasopharynx $\left({ }^{*}\right)$, which extension to across midline and caused obliterate the circumferential fat plane of right ICA more than half of diameter (arrow). Comparisons to the left sided, which preserves the normal fat plane.

(c) Axial contrast-enhanced CT scan of the nasopharynx in a different patient with NPC. The enhancing tumor mainly occupies at left nasopharynx and across midline to the right side $\left({ }^{*}\right)$, cause obliterate the circumferential fat plane of both ICA more than 180 degrees (arrow). Narrowing of left ICA is also noted. 
Fig. 6 A 57 years man known case of right nasopharyngeal cancer

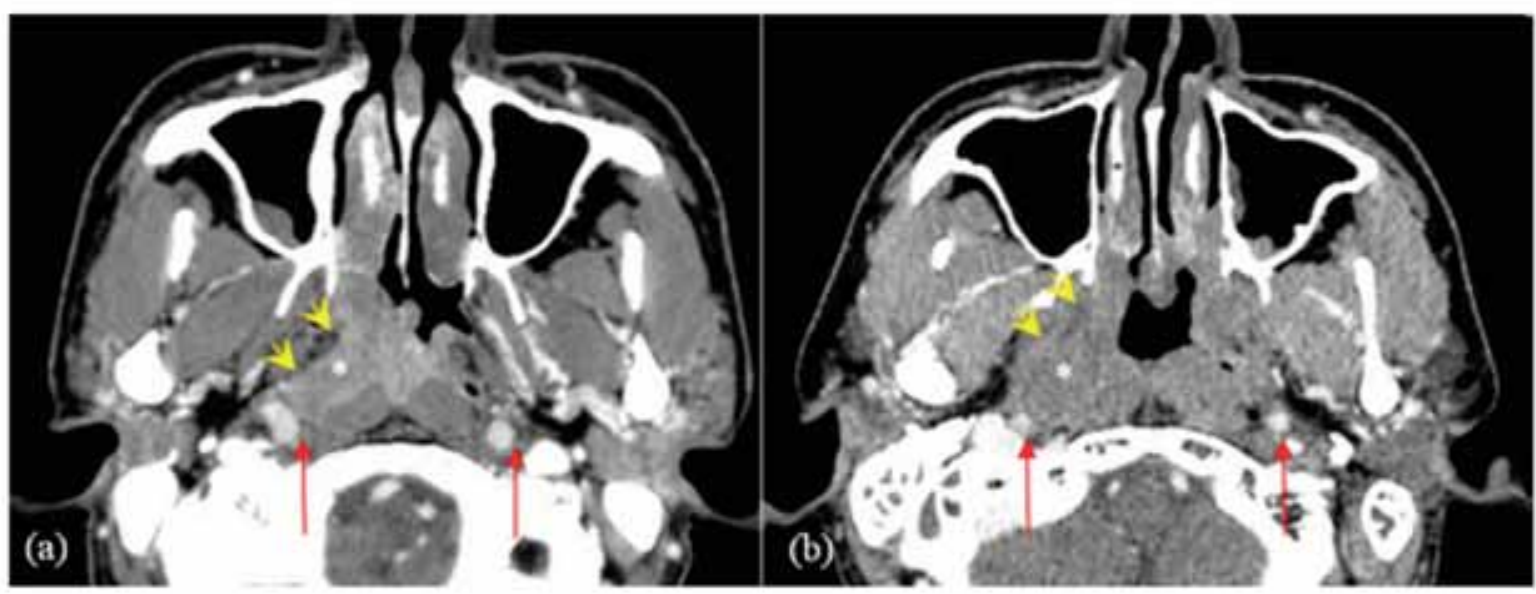

(a) Axial contrast-enhanced CT scan of the nasopharynx showed enhancing NPC at right NP (*) and extension to right parapharyngeal space; PPS (arrow head) but still preserves the normal fat plane of bilateral vascular spaces (arrow).

(b) Axial contrast-enhanced CT scan of the other patient with right NPC $\left(^{*}\right)$, revealed the NPC extends to right PPS (arrow head) and encase right carotid space nearly half of its diameter, but still preserves the normal fat plane of left vascular spaces (arrow).

Fig. 7 A 57 years old man known case of right nasopharyngeal cancer

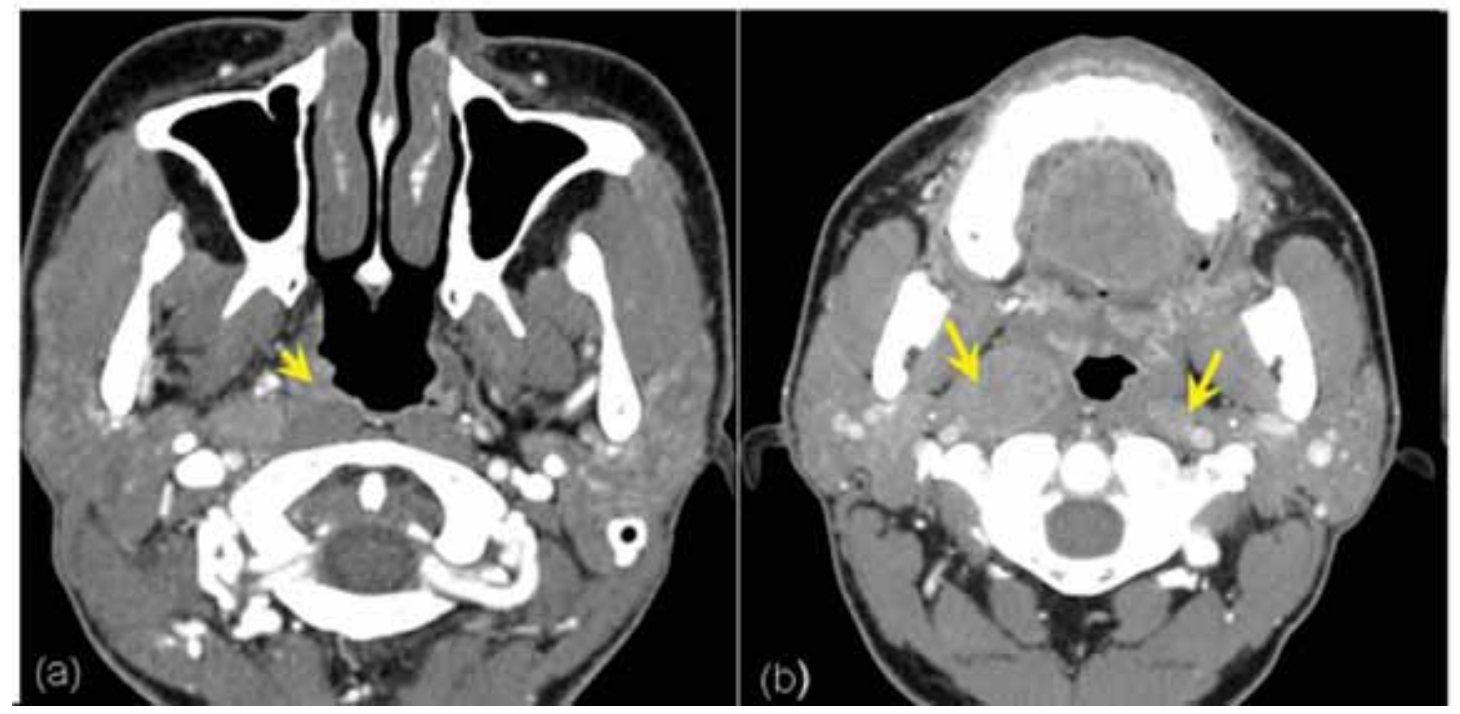

(a) Axial contrast-enhanced CT scan of this patient at level of nasopharynx show enhancing tumor at right nasopharynx (arrow head).

(b) Axial contrast-enhanced CT scan of the same patient at lower level has shown bilateral enlarged retropharyngeal lymph nodes (arrow). 
Fig. 8 A 49 years man known case nasopharyngeal cancer

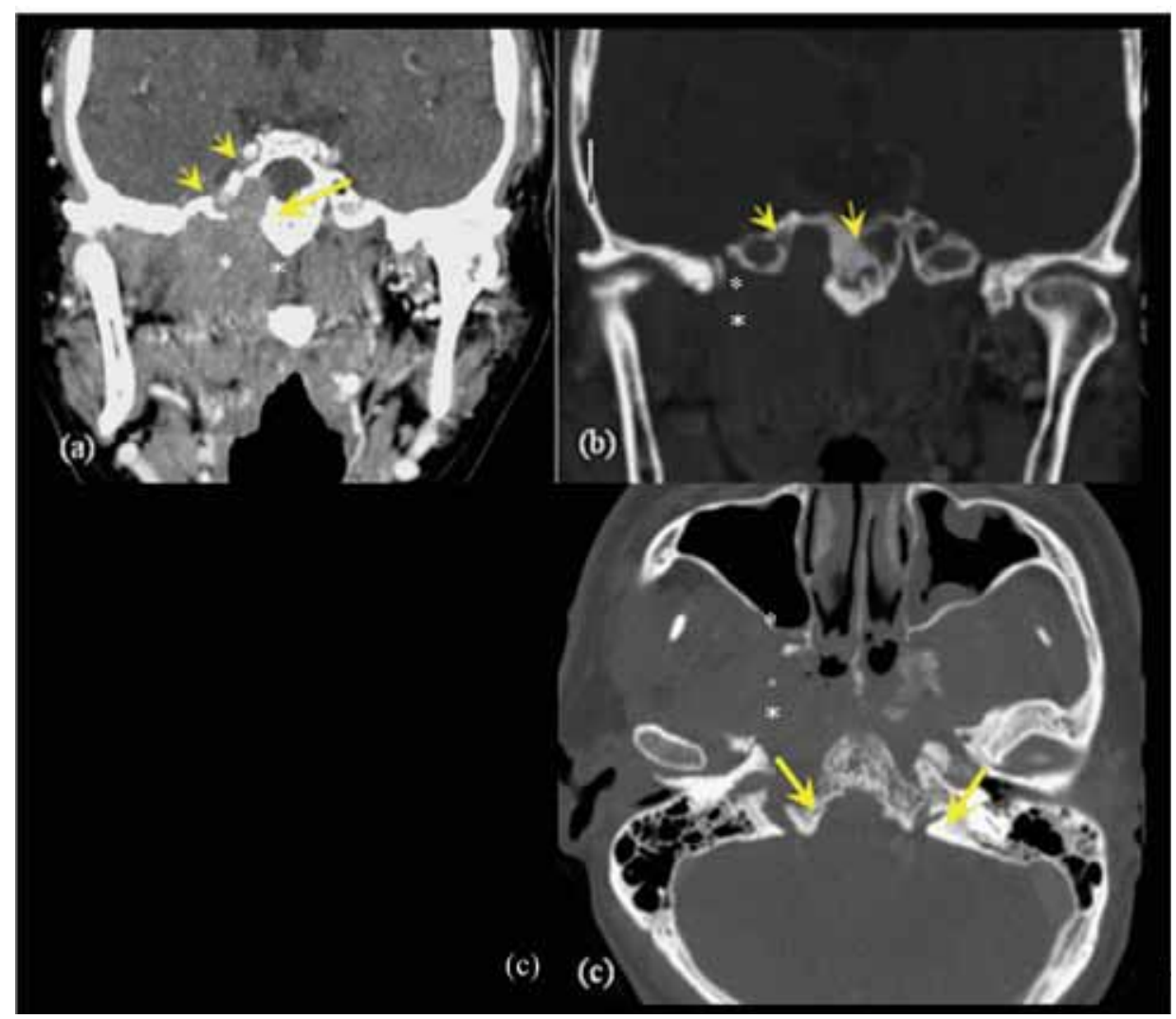

(a) Coronal view of contrast-enhanced CT scan with soft tissue window and bone window (b) shows enhancing NPC at both NP, which extended into cavernous sinus with predominate in the right sided, resulting of bony destruction at right petrous, right pterygoid plate and clivus.

(b) Axial view of contrast-enhanced CT scan with bone window showed extensive bony destruction of petrous bones, pterygoid plates, clivus and widening of both foramen lacerum, foramen ovale and foramen spinosum.

\section{Discussion}

Local recurrence and distant metastasis are the common mode of treatment failure of a nasopharyngeal cancer, which are determinant of survival. Locoregional control of NPC remains a significant factor of local recurrence. It has been accepted that the primary tumor, nodes and metastases (according to the TNM staging) is the most important prognostic factor. Different patterns of treatment failure had been defined across stages. T3-4 No-1 mostly has local failure; any $\mathrm{T}$ with $\mathrm{N} 2-3$ has risk of locoregional and distant failure ${ }^{13}$. In addition, some investigators suggested that tumor volume had a close relationship with survival rates and recurrence rates. The NPC with large tumor volume was associated with more recurrence and poor survival rate ${ }^{14}$. Moreover, De-hong et al had described the relationship between post-radiation changes and recurrent diseases and suggested, that If the nasopharyngeal walls remained moderately thick (greater than $3 \mathrm{~mm}$ ) on a CT scan beyond 6 months after radiotherapy, the risk of relapse would increase15. In our study, which initially excluded metastases, by using univariate analyses primary tumors, nodes, skull base erosion, and carotid space invasion were independent risk factors of tumor 
recurrence. According to TNM staging, the skull base involvement was corresponding to T4 stage. In term of prognostic factors, our results were in accordance with various studies, in which the higher $T$ stage had increased risk of local failure with consequently increased risk of recurrence ${ }^{16}$. However, in our data tumor size had no statistically significant correlation with the risk of recurrence. This finding was disagreed with those reports which promoted the value of tumor volume in predicting local control. This might imply that the tumor size which was measured on the single axial image was not represented the tumor volume. Although, volumetric analysis of the primary tumor has been shown to be an important prognostic value in predicting local control, but tumor volume measurement techniques is complicated and less applicable in routine work. Furthermore, NPCs tend to be infiltrative and irregularly resulting to difficult to assessment. Accurate measurement of tumor volume requires a detailed outlining of the tumor extent from imaging, and a calculation of tumor volume from a threedimensional perspective ${ }^{17}$.

For early detection of skull base erosion, multidetector CT scan has been the reliable and the most cost-effectiveness imaging tool for evaluation. MRI should be performed when subtle lesions adjacent to the skull base or subtle intracranial invasion is suspicious. Comparison the capacity between CT scan and MRI was not the purpose of this study.

Carotid space invasion had been recognized which showing significant correlation with tumor recurrence in our analysis. Generally speaking, carotid space is post styloid compartment of parapharyngeal space. Its involvement indicates posterolateral tumor extension in the parapharyneal space which is corresponding to T2b stage of TNM staging. The parapharyngeal space was the most commonly infiltrated region.

Involvement of parapharyngeal space was shown to be associated with prognostic factor and poor local control in several reports ${ }^{16}$. KalogeraFountzila $\mathrm{A}$ et $\mathrm{al}^{16}$ demonstrated that the degree of the tumor extension into the parapharyngeal space was an independent prognostic factor for overall survival. Our data suggested that invasion of the carotid space which was indicated by loss of fat plane surrounding the internal carotid artery was more important sign than invasion of parapharyngeal space alone. To evaluate infiltration of perivascular fat plane, MRI is more accurate and preferred to be diagnostic method particularly if it is not extensive involvement, however, multidetector CT scan can be applied. Based on our result, skull base erosion and carotid space involvement had statistically significant, thus, posterior extension of the NPC may confer the worse prognosis.

The incidence of presence of cervical lymph node involvement in patients with NPC is quite high. About $75 \%$ of patients have enlarged cervical nodes at presentation ${ }^{18}, 80 \%$ are bilateral. Nodal metastasis shows an orderly inferior spread and the affected nodes are larger in the upper neck ${ }^{19}$. It was found $100 \%$ in our study. There was correlation between lymph node involvement according to TNM staging and risk of tumor recurrence $(p=0.02)$, however, the size and the nodal levels had no significant effect.

Our data revealed metastatic retropharyngeal lymph node at $39.4 \%$, but no difference between recurrent and non recurrent groups. It is considered to be primary station of NPC and may be seen in $65 \%$ of patients with cervical lymph node metastases, $35 \%$ of metastasis bypasses this node to reach the 
cervical nodes ${ }^{20}$. Some authors had also reported that the distant failure for these lymph node metastases was similar to level II lymph node involvement. In the staging system of NPC, involvement of retropharyngeal lymph node has not been mentioned routinely. How its abnormality influence on prognosis of NPC is still not well understood. The reports of no significant influence of retropharyngeal lymph node on predicting outcome ${ }^{21}$ are in agreement with our results.

Concerning the lymph node size, although we found that the averaged diameter of the largest lymph node of recurrent group was larger than the other group (3.6 cm and $1.5 \mathrm{~cm}$ respectively) but it was no significant difference ( $p$ valve $<0.05$ ) in relation to the risk of recurrence. However, there has been controversy over the prognostic significance of the size and various features of lymph node involvement. Lee et $\mathrm{al}^{22}$ found that maximum lymph node size was independently significant in predicting survival, but in contrast, many investigators found that it was not a significant prognostic factor ${ }^{23,24}$. Furthermore, Teo et $\mathrm{al}^{23}$ reported that fixed lymph nodes, and contralateral neck lymph nodes were independently significant in predicting survival, rather than maximal lymph node size.

Among the other factor that were significant, only skull base invasion had been identified to be an independent factor for prediction of NPC recurrence in multivariate analysis. However, those findings may be correlated with one another. Because of late local recurrences, even after five years are not rare, and those patients who had developed distant metastases after primary radiotherapy had a better prognosis ${ }^{22}$, thus, early detection of recurrent disease or distant metastases after complete treatment is very important. For NPC patients who have those suggestive findings, particularly, skull base invasion should be followed up closely.

\section{Conclusion}

The risks of NPC recurrence after complete treatment have been correlated to TNM staging, skull base invasion, and carotid space involvement. Neither tumor size, lymph node size, parapharyngeal space invasion alone nor retropharyngeal lymph nodes have been shown to be significant prognostic factors. Skull base invasion has been identified to be the predictive sign for tumor recurrence. Skull base invasion and carotid space involvement have a statistically significant prognostic factors for overall survival. Carotid space involvement is indicated by infiltration of perivascular fat plane of the internal carotid artery.

\section{Acknowledgements}

I would like to express the deepest appreciation to my committee members, Professor Anchalee Churojana, M.D. and Kullathorn Thephamongkhol, M.D. who have the attitude and the substance of a genius: they continually and convincingly conveyed a spirit of adventure in regard to research, and an excitement in regard to teaching. Without their guidance and persistent help this dissertation would not have been possible.

I would like to thank to advisor Chulalak, who was my statistically counseling, all nursing staffs and helpers in diagnostic radiology and radiotherapy departments at Siriraj hospital who generously dedicated their times and efforts on this important work piece. Without them, I would spend a decade to master my dissertation.

Lastly, the important to the success of my research also are my dad, mom, sisters, my friends, 
and overall radiology residents who have supported my work. I thank you all from the bottom of my heart.

\section{Umasan Phuprasat, M.D.}

\section{References}

1. Parkin DM, Bray F, Ferlay J, Pisani P. NP Screen(r) Assay Risk Assessment for Nasopharyngeal Carcinoma (NPC). Global Cancer Statistic, 2002. Cancer J Clin. 2005 MarApr: 55(2);74-108.

2. Chang ET and Hans-Olov Adami. Review The Enigmatic Epidemiology of Nasopharyngeal Carcinoma. Cancer Epidemiology Biomarkers Prev. 2006(10) October:15.

3. Nguyen $\mathrm{MQ}$, Nguyen $\mathrm{CH}$, Parkin DM. Cancer Incidence in Ho Chi Minh City, Viet Nam (1995-1996). Int J Cancer: 1998;76:472-9.

4. LUO De-hong, ZHOU Chun-wu, Er-ni LI and WEN Bi-xiu. Post radiation $\mathrm{CT}$ changes and recurrent nasopharyngeal carcinoma. Chinese Medical Journal 2008;121(10):91622.

5. Comoretto et al. Detection and Restaging of Residual and/or Recurrent Nasopharyngeal Carcinoma after Chemotherapy and Radiation Therapy: Comparison of MR Imaging and FDG PET/CT: Radiology. 2008 October: 249(1).

6. สถาบันมะเร็งโรงพยาบาลศิริราช (Siriraj cancer center) www.si.mahidol.ac.th/th/department/cancer/dept history.asp-11k:

7. Isles M.G, McConkey and Mehanna H.M. A systematic review and meta-analysis of the role of positron emission tomography in the follow up of head and neck squamous cell carcinoma following radiotherapy or chemoradiotherapy. Clinical Otolaryngology 2008;33(3): 210-22.

8. Carlos Suárez, Juan P, Rodrigo, Alessandra Rinaldo, Johannes Langendijk A et al. Current treatment options for recurrent nasopharyngeal cancer: Eur Arch Otorhinolaryngol (2010);267:1811-24.

9. Teo PM, Kwan WH, Lee WY, Leung, Johnson PJ. Prognostic factors Determining Survival Subsequent to Distant Metastasis from Nasopharyngeal Carcinoma Clinical Oncology Department, Prince of Wales Hospital, Shatin, and Hong Kong Cancer. 1996 Jun 15;77(12):2423-31.
10. Ragab S.M, Faerfan, Makhalifa. The Journal of Laryngology and Otology. 2008 March; 27(22):1230-4.

11. Cooper J, Fleming I.D and Henson D.E. American Joint Committee on Cancer manual for staging of cancer (6th ed.), JB Lippincott, Philadelphia (2002).

12. Frederick L. Greene et al. 1977 AJCC nodal classification, American Joint Committee on Cancer manual for staging of cancer (6th edition), JB Lippincott, Philadelphia (2002).

13. Wei WI, Chua DT, Sham JS, Ho WK, Au GK et al. Current Management Strategy of Nasopharyngeal Carcinoma. The predictive value of the 1997 American Joint Committee on Cancer stage classification in determining failure patterns in nasopharyngeal carcinoma. Cancer (2001): 92; 2845-55.

14. Jia-Yin Zhou, Vincent F. H. Chong, James B. K. Khoo, Kap-Luk Chan, Jing Huang. The relationship between nasopharyngeal carcinoma tumor volume and TNM Tclassification: a quantitative analysis. Eur Arch Otorhinolaryngol (2007) 264:169-74.

15. LUO De-hong, ZHOU Chun-wu, LI Er-ni and WEN Bi-xiu. Post-radiation CT changes and recurrent nasopharyngeal carcinoma. Chin Med J 2008;121(10):916-22.

16. Anna Kalogera-Fountzila, Dimitrios Karanikolas, Nikos Katodritis, Epaminodas Samantas, Apostolos Sarafopoulos, Ippoliti Ikonomou et al. Prognostic Factors and Significance of the Revised $6^{\text {th }}$ Edition of the AJCC Classification in Patients with Locally Advanced Nasopharyngeal Carcinoma. Strahlenther Onkol 2006;182:458-66.

17. Jeong-Hyun Kim and Joon-Kyoo Lee. Prognostic Value of Tumor Volume in Nasopharyngeal Carcinoma. Yonsei Medical Journal 2005 (46):2;221-7.

18. Chong VF, Fan YF. Skull base erosion in nasopharyngeal carcinoma: detection by CT and MRI. Clin Radiol. 1996; 51:625-31.

19. Sham JST, Wei WI, Kwan WH, Chan CW, Kwong WK, Choy D. Nasopharyngeal carcinoma: Pattern of tumor regression after radiation. Cancer 1990;65:216-22.

20. Chong V. F. H., Fan J. B. K, and Khoo. Retropharyngeal lymphadenopathy in nasopharyngeal carcinoma. European Journal of Radiology December 1995 (21),2:15; 100-5.

21. Li-Zhi Liu, Guo-Yi Zhang, ChuangOMiao Xie, Xue-Wen Liu, Chun-Yan Cui et al. MRI of retropharyngeal node 
January-April 2013, Volume XIX No.I

metastasis in nasopharyngeal cancer: pattern of spread. Radiation Oncology Biol. Phys. 2006 (66), No. 3; 721-30.

22. Lee WY, Teo PM, Kwan WH, Leung SF, Johnson PJ. Prognostic factors Determining Survival Subsequent to Distant Metastasis from Nasopharyngeal Carcinoma Clinical Oncology Department, Prince of Wales Hospital, Shatin, Hong Kong. Clinical Onco (1996) Jun 15; 77(12):2423-31.
23. Chen HH, Prevost TC, and Duffy SW. Prognostic Factors in 677 Patients in Singapore with Nondisseminated Nasopharyngeal Carcinoma. Cancer, November 15, 1999 (86):1912-1920

24. Sham JST, Wei WI, Kwan WH, Chan CW, Kwong WK, Choy D. Nasopharyngeal carcinoma: Pattern of tumor regression after radiation. Cancer, 1990;65;216-22. 